\title{
Structure of FUN Factors in the Interaction with Products
}

\author{
Sayoko Tominaga ${ }^{1}$, Toshihisa Doi ${ }^{1}$, Toshiki Yamaoka ${ }^{2}$, \\ Yuka Misyashita $^{3}$, and Masayoshi Toriumi ${ }^{3}$ \\ ${ }^{1}$ Graduate School of Systems Engineering, Wakayama University \\ ${ }^{2}$ Faculty of Systems Engineering, Wakayama University \\ ${ }^{3}$ Vehicle Test Technology Development Division, NISSAN MOTOR CO., LTD, \\ 930, Sakaedani, Wakayama City, Wakayama, 640-8510, Japan \\ s105032@sys.wakayama-u.ac.jp
}

\begin{abstract}
In recent years interaction design has looked to questions of most typically positive emotions such as satisfaction, pleasure and delight. This study investigated the factors of FUN as joy and pleasure, which are created in interaction between human and products, and found their relationship. We aim for gaining useful information when we design products. First, the questionnaire regarding SCT (sentence completion test) were conducted and were analyzed by DEMATEL method (Decision MAking Trial \& Evaluation Laboratory) As a result, it was found that the factors of FUN were clarified and examined the relationship.
\end{abstract}

\section{Introduction}

Lately, not only Usability but also the promotion of users' psychological requirement (pleasure and joy) is needed in product development. Ministry of Economy, Trade and Industry has designated the three years from 2008 to FY 2010 as the "Kansei Value Creation Years and intensively implement a variety of measures to carry out the "Kansei Value Creation" [1]. Therefore, in Japan, "Kansei" would become an increasingly thing in product development.

In this study, the factors of FUN, as joy and pleasure, which are created in interaction between human and products, were investigated and found their relationship. These results would be useful information when we design several products.

Before introducing our full study, the area of our study is explained with the idea of "Hedonomics" [2] In conventional Ergonomics, human-centered design had been practiced to satisfy some requirements (e.g., safety, Functionality and usability). However, these were not enough requirements for users to satisfy their needs. Then, the idea of Hidonomics has come (fig.1). Hidonomics aims for the promotion of pleasure and joy in user interaction with safety, functionality and usability. In addition, it is said that Hidonomics can completely satisfy user requirements by individualized customizing. Figure 1 derived from Maslow's conception. The fact that these design imperatives match the social edict of "life, liberty and the pursuit of happiness" has not escaped our attention (see Hancock, 1999). In this study, "FUN" is defined the interaction factors which roused pleasure and joy to create "Pleasurable Experience" in the idea of Hedonomics. 
"Kansei" which was used this paper means a sequence of information flow (sense $\rightarrow$ awareness/cognitive $\rightarrow$ emotion/affectivity $\rightarrow$ the expressions of language) which occur after exterior stimulus is communicated to sensory receptor.

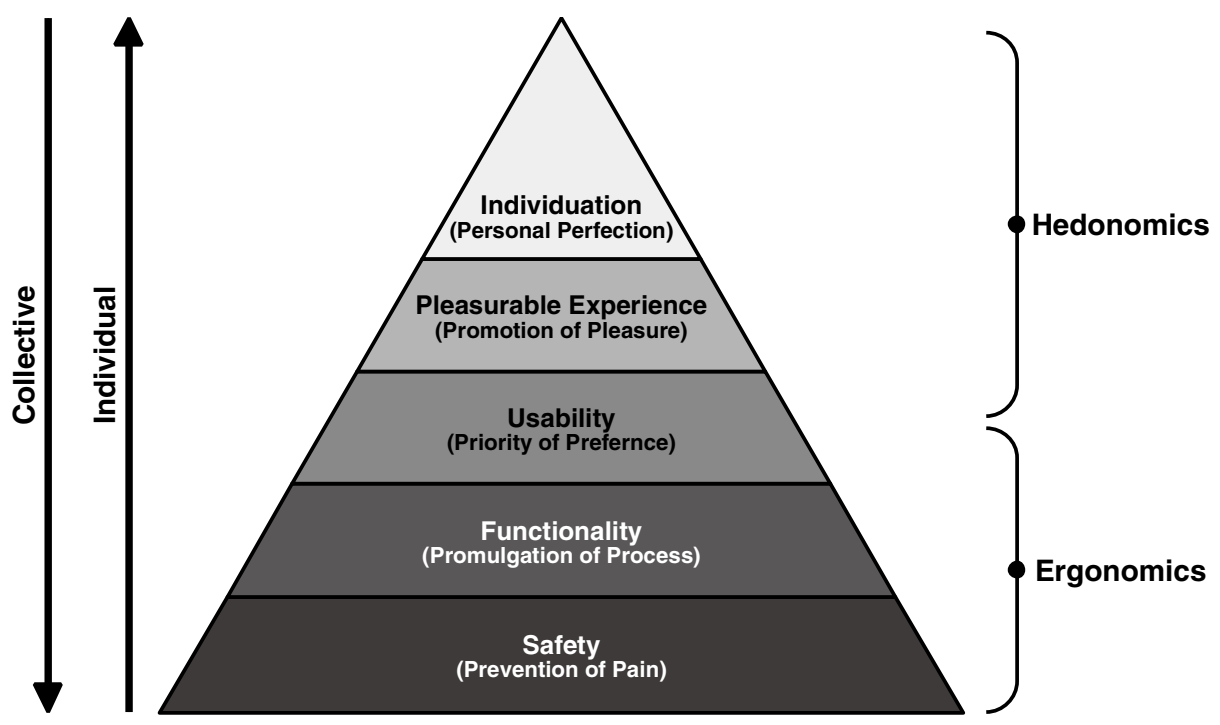

Fig. 1. A hierarchy of the relationship between Ergonomics and Hedonomics [2]

\section{Conducting a Questionnaire}

\subsection{Summary of a Questionnaire}

This study investigated the factors of FUN and found their relationship. We aim for gaining useful information when we design products. First, the questionnaire regarding SCT (sentence completion test) were conducted and the results of the questionnaire is analyzed by DEMATEL method (Decision MAking Trial \& Evaluation Laboratory) To investigated the factors of FUN and their relationship, SCT by a questionnaire was conducted to some students and workers. We will grasp the factors of FUN which human generally feels from products and analyze their relationship by DEMATEL method.

\subsection{Participants}

Study participants included 33 adults from twenty-two to forty-nine. (Average: 31, SD:9.38, man:24, woman:9) It is very difficult for ordinary people to answer our questionnaire. Therefore, in this study, we confined the participants to only users who know HMI (Human Machine Interface), product design, or user interaction design. In doing so, we concerned that better answer can be gotten. 


\subsection{Contents of the Questionnaire}

SCT is one of the Projective Technique method. SCT is a method that participants are asked to fill in the blanks on the sentence to complete with the words which they thought. [3]. The Projective Technique method is a method that participants are showed ambiguous stimulus and asked to interpret it and to explain it. The more ambiguous the stimulus is, the more participants tend to express their hidden viewpoint and emotion in one way or another. Therefore, this method aims to have participants express their genuine thought.

In this study, we conducted the following questionnaire to find the factors of FUN. The question is "Please show me the products which make user feel pleasure and joy through the interaction between products and users?" Participants are asked to fill in the blanks on the sentence which shows "Because ( ), ( ). Then, ( )." with their thought. Figure 2 shows a questionnaire actually used in this study.

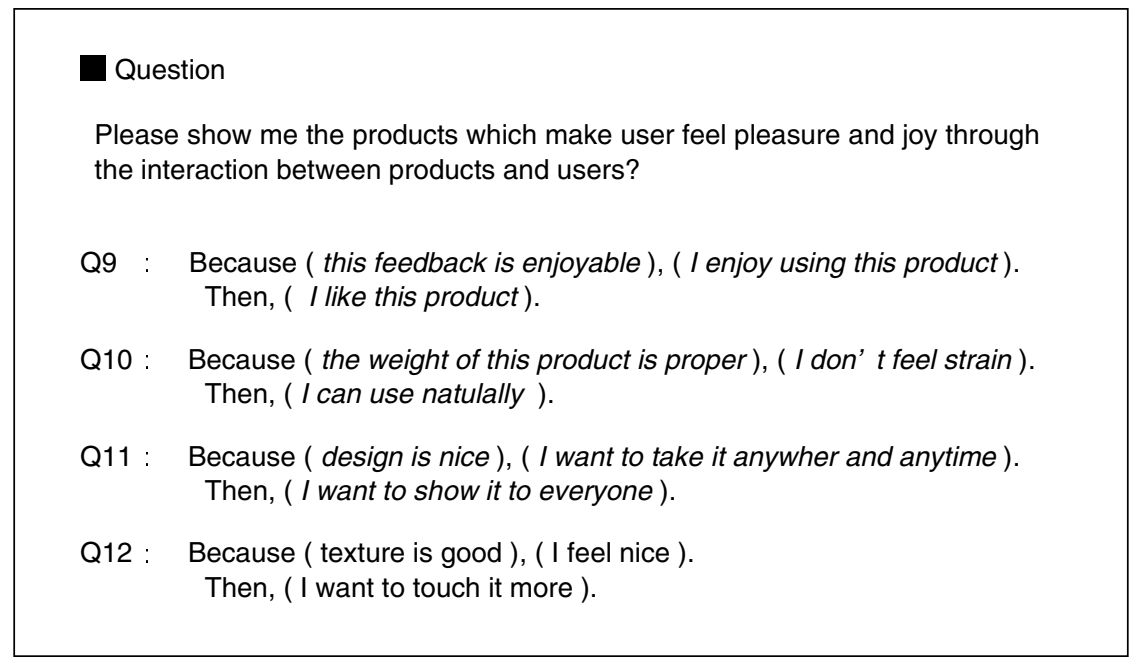

Fig. 2. Questionnaire of SCT

\section{Results of SCT}

To visualize the FUN factor's relationship, data obtained from SCT were analyzed with DEMATEL method. DEMATEL method was developed to grasp the difficult and complicated problem structure. As its feature, the method can represent not only the existence of factors' relationship but also the strength of their relationship among factors.

Before starting to analyze, the matrix needed the DEMATEL method was made from gained sentence as explained next. First, words and phrases gained from a questionnaire were coded and made a classification of the coded words on the basis of similar significance. Second, the keywords from one sentence were divided into cause and effect and two causations were made (fig3). (Ex: "Because (A), (B). Then, (C)." was changed into "A-B" and "B-C"). Finally, an $n$ times $n$ matrix is made from the degree of the each causation (Fig4). 
(1) The sentence gained from a questionnaire

Because $(A),(B)$.Then, $(C)$.

(2) Devided into two causal pairs from one sentence

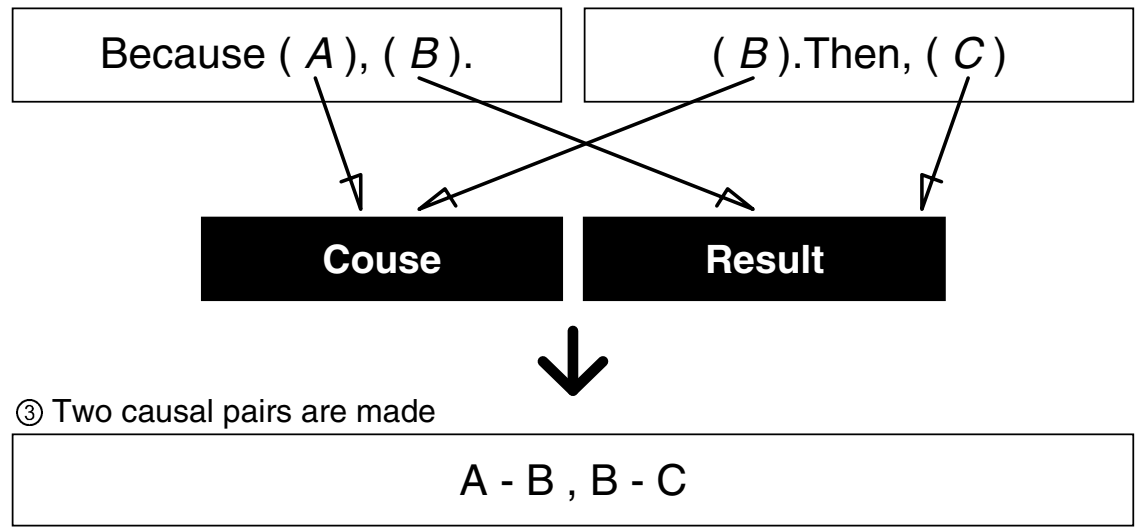

Fig. 3. How to make two causations from one sentence

\begin{tabular}{|c|c|c|c|c|c|c|c|c|}
\hline & \multicolumn{6}{|c|}{ Keyword } & \multirow{2}{*}{ [ D ] } \\
\hline & & A & B & C & D & $E$ & $\mathrm{~F}$ & \\
\hline \multirow{6}{*}{ 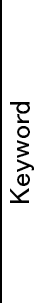 } & A & 0 & 1 & 1 & 3 & 0 & 0 & 5 \\
\hline & B & 2 & 0 & 0 & 0 & 1 & 0 & 3 \\
\hline & C & 0 & 0 & 0 & 0 & 1 & 1 & 2 \\
\hline & D & 1 & 1 & 3 & 2 & 0 & 0 & 7 \\
\hline & $E$ & 0 & 0 & 0 & 1 & 1 & 0 & 2 \\
\hline & $F$ & 0 & 2 & 1 & 1 & 1 & 0 & 5 \\
\hline & [ R ] & 3 & 4 & 5 & 7 & 4 & 1 & 24 \\
\hline
\end{tabular}

Fig. 4. Matrix to analyze with the DEMATEL method (sample)

The row sum D of the matrix represents the total sum of influence level (influence rate) from a certain keyword to another keyword, while the rank sum $\mathrm{R}$ of the matrix represents the total sum of influenced level (influenced rate) from a certain keyword to another keyword.

D plus R means "Degree of association". "Degree of association" represents how much the keyword plays a key role in this structure of the problem because $\mathrm{D}$ plus $\mathrm{R}$ is the sum of the influence rate and influenced rate. On the other hand, D minus $\mathrm{R}$ represents the difference of the two. D minus R means "Influence rate". "Influence rate" represents how much the keyword influence others keywords as a cause. If this 
value is plus, it shows that the keyword influence another keywords. If this value is minus, it shows that the keyword is influenced from other keywords.

Additionally, we conducted the hierarchical clustering to consider the relationship among gained keywords from their Degree of association and Influence rate. Gained keywords were divided into five clusters. As a result, it found that Cluster A has five keywords, Cluster B has eleven keywords, Cluster C has twenty-one keywords, Cluster D has thirteen keywords and Cluster E has eight keywords. The following figure which is shown the Degree of association as $\mathrm{x}$-axis and the Influence rate as $\mathrm{y}$-axis represents every gained keywords plotted.

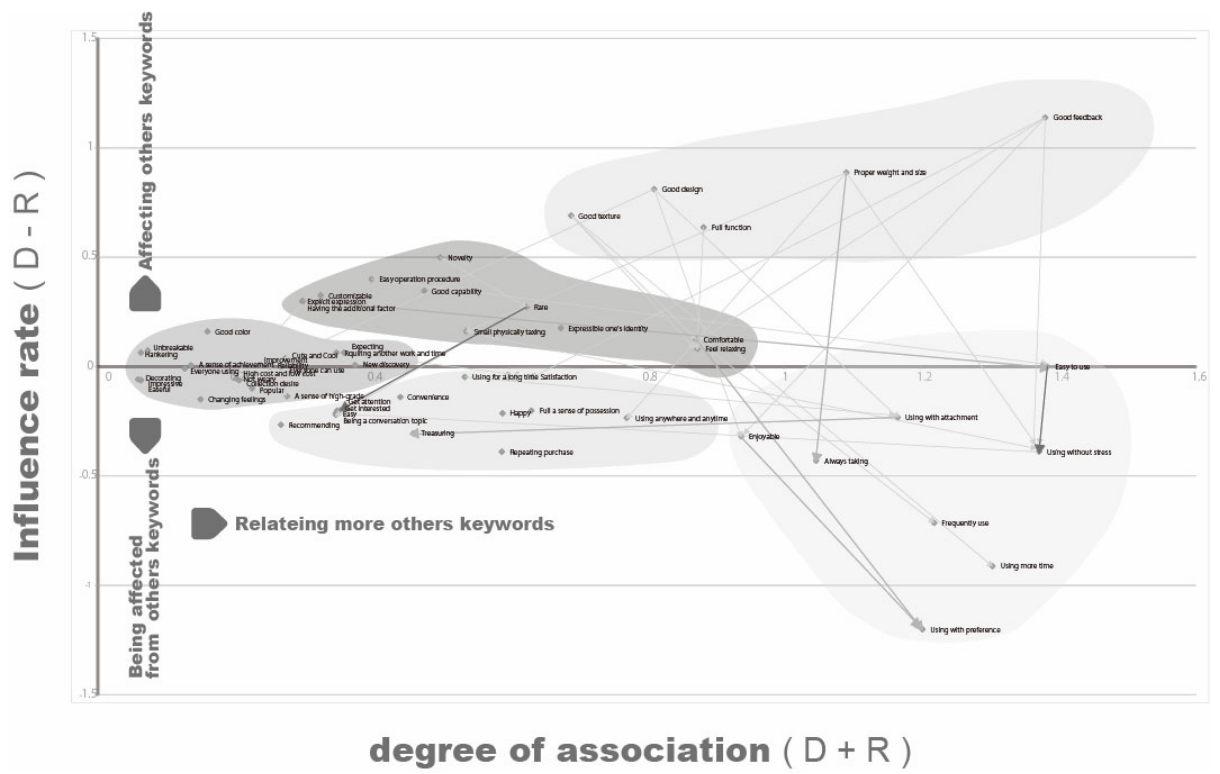

Fig. 5. Visualization of the result of the DEMATEL method

\section{Discussion}

We will explain each group classified into five clusters. Then, we will discuss structure as a whole.

Cluster A is a cluster which has high Degree of association and high Influence rate. In other words, each words of Cluster A relate more others keywords and affect them. The words regarding "Product's attribution" were seen from Cluster A. They are "Good feedback", "Good texture", "Proper weight and size",'Good design" and "Full function".

Cluster B is a cluster which has some Degree of association and Influence rate but both of numbers of Cluster B are not higher than those of Cluster A. In other words, each words of Cluster B relate some keywords and affect them. The words regarding "Concrete usability and convenient" were seen from Cluster B. They are "Novelty", "Customizable", "Explicit expression", and "Comfortable". 
Cluster $\mathrm{C}$ is a cluster which Degree of association is the lowest of the five clusters. Influence rate of Cluster $\mathrm{C}$ is neither low nor high. In other words, each words of Cluster $\mathrm{C}$ are individual solution so that they don't particularly relate other keywords. Additionally, it seems that Cluster $\mathrm{C}$ is a neutral position cluster because their words have the possibility to affect other words and to be affected from others words. The words regarding "Emotion" were seen from Cluster C. They are "Changing feelings", "Hankering", "Reliability", and "Impressive". It is considered that Influence rate of Cluster $\mathrm{C}$ is the lowest so that emotion is the personal factor.

Cluster D is a cluster which Degree of association is as much numbers as Cluster B and Influence rate is second lowest cluster of the five. In other words, Cluster D is a cluster which is affected from other clusters. The words regarding "Relation to other people" were seen from Cluster D. They are "Get attention", "Being a conversation topic", "Recommending", "Satisfaction" and "Using for a long time". Cluster D is affected from Cluster A or Cluster B. For this reason, it seems that Cluster D also means a people's next action which will occur in filling situation of Cluster A or Cluster B.

Cluster $\mathrm{E}$ is a cluster which Degree of association is as high numbers as Cluster A and Influence rate is the lowest cluster of the five. In other words, each words of Cluster E relate more other keywords and are affected from other keywords. The words regarding "Frequency of use and Status of use" were seen from Cluster E. They are "Using with preference", "Using more time", "Using without stress", "Using with attachment" and "Always taking".

Next, we will explain the whole causal relation. According to each discussion, it found that the concept starts Cluster A (Product's attribution) as high conception and connects to Cluster B (Concrete usability and convenient) and next connect to Cluster $\mathrm{C}$ (Emotion) and Cluster D (Relation to other people), then, last connects to Cluster E (Frequency of use and Status of use). For this reason, it would appear the following discussion. The enchantments of product's attribution give good Usability to user. Next, positive emotion occurs to users and users try to relation to other people. Finally, it happened that frequency of use becomes more and the tendency of use becomes more positive.

As another consideration, it found that the top 3 keywords which have high Degree of association are the keywords regarding usability. For instance, "Easy to use", "Good feedback" and "Using without stress". For this reason, it is again found the view of usability is very important for people to feel pleasure and enjoyment on interaction between human and products.

\section{References}

1. Ministry of Economy, Trade and Industry: Kansei Value Creation Initiative (2007), http: / /www.meti.go.jp/press/20070522001/20070522001.html

2. Hancock, P., Pepe, A., Murphy, L.: Hedonomics: The Power of Positive and Pleasurable Ergonomics, p. 11. Human Factors and Ergonomics Society (2005)

3. Usability Handbook editorial committee: Usability Handbook. Kyouritsu-Pub. Co., p. 686, p. 564 (2007) 